\title{
We-Measure: Toward a low-cost portable posturography for patients with multiple sclerosis using the commercial Wii balance board
}

\author{
Letizia Castelli $^{\text {a }}$, Luca Stocchi ${ }^{\text {b }}$, Maurizio Patrignani ${ }^{\text {b }}$, Giovanni Sellitto ${ }^{\text {a }}$, Manuela Giuliani ${ }^{\mathrm{c}}$, Luca Prosperini ${ }^{\mathrm{c}, *}$ \\ a Physical Therapy Unit, S. Andrea Hospital, Sapienza University, Rome, Italy \\ b Department of Engineering, Roma Tre University, Rome, Italy \\ c Department of Neurology and Psychiatry, Sapienza University, Rome, Italy
}

\section{A R T I C L E I N F O}

\section{Article history:}

Received 11 August 2015

Received in revised form 17 September 2015

Accepted 8 October 2015

Available online $\mathrm{xxxx}$

\section{Keywords:}

Multiple sclerosis

Force platform

Static posturography

Balance board

Wii

\begin{abstract}
A B S T R A C T
This study was aimed at investigating whether postural sway measures derived from a standard force platform were similar to those generated by a custom-written software ("We-Measure") acquiring and processing data from a commercial Nintendo balance board (BB). For this purpose, 90 patients with multiple sclerosis (MS) and 50 healthy controls (HC) were tested in a single-day session with a reference standard force platform and a BB-based system.

Despite its acceptable between-device agreement (tested by visual evaluation of Bland-Altman plot), the lowcost BB-based system tended to overestimate postural sway when compared to the reference standard force platform in both MS and HC groups (on average $+30 \%$ and $+54 \%$, respectively). Between-device reliability was just adequate (MS: 66\%, HC: 47\%), while test-retest reliability was excellent (MS: $84 \%$, HC: $88 \%$ ). Concurrent validity evaluation showed similar performance between the reference standard force platform and the BB-based system in discriminating fallers and non-fallers among patients with MS. All these findings may encourage the use of this balance board-based new device in longitudinal study, rather than in cross-sectional design, thus providing a potential useful tool for multicenter settings.
\end{abstract}

(c) 2015 Elsevier B.V. All rights reserved.

\section{Introduction}

Computer-based estimations of postural sway by means of force platform have been recently suggested as reliable, objective and specific measures for balance in patients with multiple sclerosis (MS) [1-5].

However, despite its theoretical advantages, the lack of standardization, heterogeneity of equipments and expensiveness make the use of force platforms not easily feasible in daily clinical setting, and unsuitable for multicenter research purposes [1]. A possible solution to overcome these drawbacks may be the implementation of software to interface a commercial balance board (BB) of the Nintendo Wii system with a common personal computer [6-9]. Similarly to laboratory-grade force platforms, the BB contains cells which detect body's load shifts, potentially providing an alternative method to perform a static posturography assessment $[1,9]$.

Literature data suggest that BB-based outcomes of balance may be considered as a reliable and useful tool to quantify postural sway in general population, older people, stroke survivors and visually impaired patients $[6,10-14]$. However, despite the growing interest about the

\footnotetext{
* Corresponding author at: Department of Neurology and Psychiatry, Sapienza University, Viale dell'Università, 30 - 00185 Rome, Italy.

E-mail address: luca.prosperini@uniroma1.it (L. Prosperini).
}

possible applications of this commercial device in medical and rehabilitation settings, studies exploring the role of BB-derived measures of postural sway in patients with MS are still lacking. Therefore, in this study we aimed at investigating whether measurements of postural sway provided by the commercial BB (cost: about 80 euros) were similar to those derived from a standard force platform (cost: more than 5000 euros) in patients with MS and healthy control (HC) subjects.

\section{Methods}

\subsection{Participants}

Patients with MS regularly attending the outpatient MS Centre of S. Andrea Hospital in Rome and a convenience sample of HCs were consecutively recruited. To be eligible, patients were required to have: an age from 18 to 65 years; clinical stability from at least three months; ability to stand upright for at least $180 \mathrm{~s}$ without any support; voluntarily provide a valid informed consent before any study procedure. Exclusion criteria were: exacerbations in the previous three months; current use of oral/systemic corticosteroids; initiation of disease-modifying or symptomatic treatments, or any medication change occurring over the last months; severe cognitive impairment or any other clinically relevant medical condition that could interfere with the study protocol. 
A

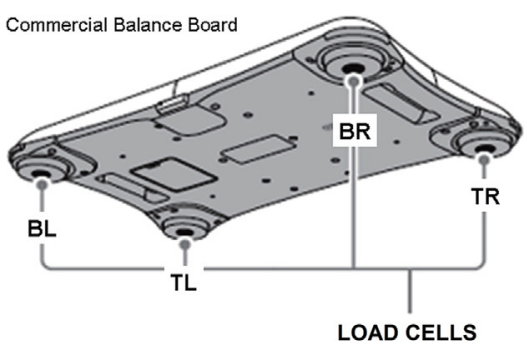

B

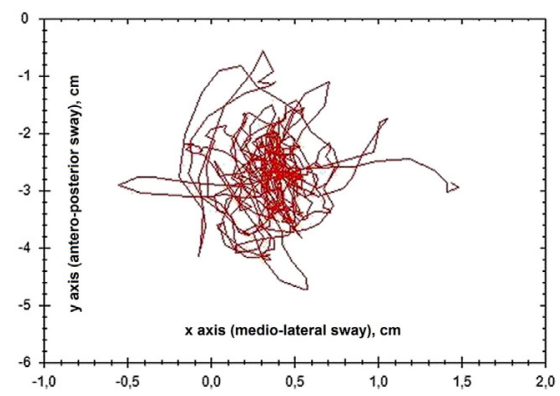

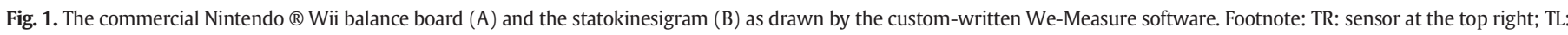
sensor in the top left; BR: sensor in the bottom right; BL: sensor on the bottom left.

Patients with MS were also asked to retrospectively report the occurrence of accidental falls (i.e. an unexpected contact of any part of the body with the ground) in the past $1,3,6$ or 12 -month period.

\subsection{Study procedures}

All participants were tested by means of a "reference standard" force platform (ProKin PK-254P, Tecnobody, Bergamo, Italy) and a BB-based system.

The ProKin PK-254P is a unipedal proprioceptive platform designed for rehabilitation purposes which includes also a monoaxial force platform (consisting of 3 strain gauges set in a triangular position under a surface of $55 \mathrm{~cm}$ diameter, with a $20 \mathrm{~Hz}$ sampling rate and $0.1^{\circ}$

Table 1

Characteristics of study participants.

\begin{tabular}{|c|c|c|c|}
\hline & $\begin{array}{l}\text { Patients with MS } \\
\mathrm{N}=90\end{array}$ & $\begin{array}{l}\text { Healthy volunteers } \\
\mathrm{N}=50\end{array}$ & \\
\hline Gender, n (females: males) & $59: 31$ & $33: 17$ & \\
\hline \multicolumn{4}{|l|}{ Age, years } \\
\hline mean $(S D)$ & $39.1(10.3)$ & $39.0(9.4)$ & \\
\hline median [range] & 38 [18-60] & 38 [22-59] & \\
\hline \multicolumn{4}{|l|}{ Weight, kg } \\
\hline mean $(S D)$ & $64.6(12.9)$ & $66.1(12.1)$ & \\
\hline median [range] & 63 [45-99] & $66[47-98]$ & \\
\hline \multicolumn{4}{|l|}{ Height, m } \\
\hline mean $(S D)$ & $1.67(0.09)$ & $1.68(0.08)$ & \\
\hline median [range] & $1.66[1.47-1.88]$ & $1.69[1.55-1.83]$ & \\
\hline \multicolumn{4}{|l|}{ BMI, $\mathrm{kg} / \mathrm{m}^{2}$} \\
\hline mean $(S D)$ & $22.9(4.0)$ & $23.3(3.8)$ & \\
\hline median [range] & 22.5 [15.5-38.7] & 22.6 [17.5-33.7] & \\
\hline MS phenotype, n (RR:SP) & $68: 22$ & $\mathrm{~N} / \mathrm{A}$ & \\
\hline \multicolumn{4}{|l|}{ Disease duration, years } \\
\hline mean $(S D)$ & $12.1(8.5)$ & $\mathrm{N} / \mathrm{A}$ & \\
\hline median [range] & $10[1-38]$ & & \\
\hline \multicolumn{4}{|l|}{ EDSS score } \\
\hline mean $(S D)$ & $3.0(1.5)$ & $\mathrm{N} / \mathrm{A}$ & \\
\hline median [range] & $2.5[1.0-6.5]$ & & \\
\hline \multicolumn{4}{|l|}{ PK-254P - COP path, mm } \\
\hline mean $(S D)$ & $297(140)$ & $195(69)$ & \\
\hline median [range] & $257[115-732]$ & $187[92-382]$ & \\
\hline \multicolumn{4}{|l|}{$\mathrm{BB}($ trial 1$)$ - COP path, mm } \\
\hline mean $(S D)$ & $386(130)$ & $310(70)$ & \\
\hline median [range] & $381[220-878]$ & $290[165-503]$ & \\
\hline \multicolumn{4}{|l|}{$\mathrm{BB}$ (trial 2) - COP path, mm } \\
\hline mean $(S D)$ & $403(150)$ & $301(75)$ & \\
\hline median [range] & $392[225-924]$ & $285[148-500]$ & \\
\hline
\end{tabular}

BB: balance board-based system; BMI: body mass index; COP: center of pressure; EDSS: Expanded Disability Status Scale; MS: multiple sclerosis; N/A: not applicable; PK-254P: reference standard force platform ProKin; RR: relapsing-remitting; SD: standard deviation; SP: secondary progressive.

* $p<0.001$. sensitivity). Although this device cannot be considered as a goldstandard laboratory-grade force platform (its sampling rate is below the recommended limits of $50-100 \mathrm{~Hz}$ [15]), it showed good concurrent validity, with correlation coefficients close to 1 in comparison with a triaxial force platform acquiring data at $996 \mathrm{~Hz}$ frequency [4].

The BB is shaped like a household body scale measuring $45 \times 26.5 \times 3.2 \mathrm{~cm}$; it contains four sensors positioned in the four corners, allowing to detect load shifts on its surface (see Fig. 1/A). These four load cells work at an inconstant sampling rate ranging from 30 to $50 \mathrm{~Hz}[9,16]$ to about $100 \mathrm{~Hz}$ [17].

Participants were asked to maintain their double-leg standing balance as steady as possible for $30 \mathrm{~s}$ with eyes opened to estimate the instant positions of the center of pressure (COP) on the ground to calculate the COP path length, defined as the sum of displacements (millimeters - mm) in a 30-s trial. The COP path length represents a widely accepted measure of postural sway, i.e. the constant slight corrective deviation from the vertical when standing upright [18].

The two devices were placed in two different rooms and assessments were done by two different trained operators (LC and GS) according to standardized procedures. Each participant underwent, in random order, three consecutive posturography assessments separated by a 15-min interval, once using the reference standard ProKin PK-254P and twice using the BB-based device. Simultaneous test sessions by superposing the BB to the ProKin PK-254P were not done to avoid loss of sensitivity of the bottom platform. Although our approach might have led to an increased measurement variability, previously published papers support the excellent reliability of within-day sway analysis in both general population and patients with MS [19,20].

\subsection{The "We-Measure" software}

The under investigation device included the BB, a Bluetooth-equipped laptop, and a custom-written software ("We-Measure") that was created at the Department of Engineering, Roma Tre University (see ahead for more details). A wireless communication protocol via Bluetooth technology is adopted for interfacing the balance board either with the Wii console and any other computer or laptop. Therefore, the "We-Measure" software was created for the following aims: (i) to connect the balance board with a common laptop computer via Bluetooth [by means of the WiiMoteLib library]; (ii) to calculate the COP coordinates (expressed as $\mathrm{mm}$ ) from the values recorded by the four sensors (expressed as force kgf), according to equations reported by Bartlett and coll. [17]; (iii) to show trial results, including the $\mathrm{COP}_{\mathrm{x}, \mathrm{y}}$ coordinates, the velocity of COP $(\mathrm{mm} / \mathrm{s})$, and the COP path $(\mathrm{mm})$ [by means of the System Drawing library], calculated by means of well-established formulas [21]; (iv) to display a real-time visualization of the statokinesigram (i.e. a graph depicting all the successive position of the COP during the 30s-recording) [by means of the ZedGraph library] (see Fig. 1/B). 
A

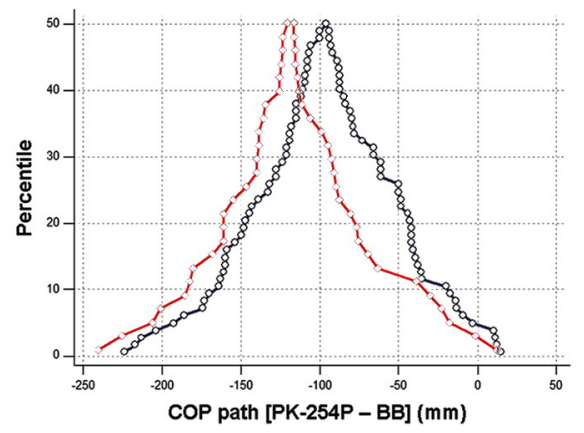

B

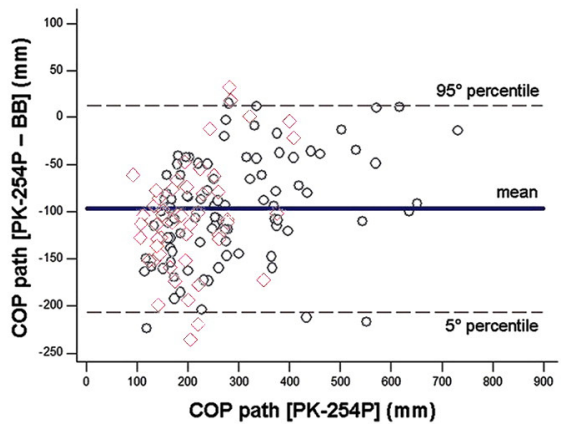

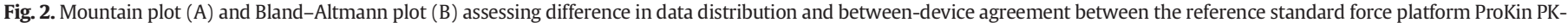

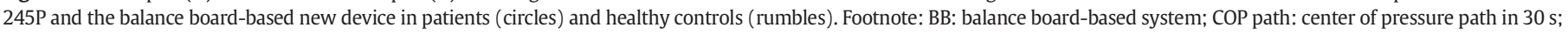
PK-254P: reference standard force platform.

To avoid the signal noise due to the high sensitivity of the four sensors, the We-Measure software provides three arrangements: (i) before starting the assessment, a calibration procedure is warranted to set to zero incorrect values that may be recorded soon after switching-on the WBBS; (ii) a low-pass filter with a cut-off sampling rate of $20 \mathrm{~Hz}$ was applied (to obtain a signal similar to that from the ProKin PK254P); (iii) less than 20-kg weights were considered equal to $0 \mathrm{~kg}$.

The freeware "We-Measure" software was created at the Department of Computer Sciences and Automation (Roma Tre University, Rome) and it is available at the following URL: http://www.dia. uniroma3.it/ patrigna/portable_post/.

\subsection{Statistical analysis}

Between-device agreement was investigated by means of BlandAltman and Mountain plots. Two-way, random-effects, single measure, intraclass correlation coefficients for absolute agreement $\left(\mathrm{ICC}_{2,1}\right)$ and standard error of measurement (SEM), calculated as SD * (square root of (1-ICC)), were also carried out to test the between-device reliability. A similar approach was done to investigate test-retest reliability of two posturography assessments performed with the BB-based new device. ICCs were considered excellent $(>0.75)$, adequate $(0.4-0.74)$, or poor $(<0.39)[22]$.

The concurrent validity of the two different systems was tested by comparing the receiver operating characteristic (ROC) curves describing the relationship between the occurrence of accidental falls and postural sway found with the reference standard PK-254P and BB-based system. Data were analyzed using a PC version of Statistical Package for the Social Sciences, version 16.0 (IBM SPSS, Chicago, IL, USA).

Table 2

Summary of study results in terms of inter-device reliability and within-device reliability.

\begin{tabular}{|c|c|c|c|c|c|}
\hline \multicolumn{6}{|c|}{ Inter-device reliability } \\
\hline & PK-254P mean (SD) & $\begin{array}{l}\text { BB } \\
\text { mean (SD) }\end{array}$ & $\begin{array}{l}\text { Difference } \\
\text { mean }(S D)\end{array}$ & $\begin{array}{l}\text { SEM } \\
\text { (95\% CIs) }\end{array}$ & $\begin{array}{l}\text { ICC } \\
(95 \% \text { CIs })\end{array}$ \\
\hline $\operatorname{MS}(n=90)$ & $297(140)$ & $386(130)$ & $89(67)$ & $82(52-123)$ & $0.667(0.258-0.872)$ \\
\hline $\mathrm{HC}(\mathrm{n}=50)$ & $195(69)$ & $310(70)$ & $105(53)$ & $66(41-82)$ & $0.467(0.173-0.796)$ \\
\hline \multicolumn{6}{|c|}{ Test-retest reliability } \\
\hline & $\begin{array}{l}\mathrm{BB}-\text { trial } 1 \\
\text { mean }(\mathrm{SD})\end{array}$ & $\begin{array}{l}\mathrm{BB}-\text { trial } 2 \\
\text { mean }(\mathrm{SD})\end{array}$ & $\begin{array}{l}\text { Difference } \\
\text { mean (SD) }\end{array}$ & SEM (95\% CIs) & $\begin{array}{l}\text { ICC } \\
(95 \% \text { CIs })\end{array}$ \\
\hline MS $(n=90)$ & $386(130)$ & $403(150)$ & $17(52)$ & $55(43-71)$ & $0.845(0.742-0.906)$ \\
\hline $\mathrm{HC}(\mathrm{n}=50)$ & $310(70)$ & $301(75)$ & $9(37)$ & $31(14-48)$ & $0.885(0.730-0.976)$ \\
\hline
\end{tabular}

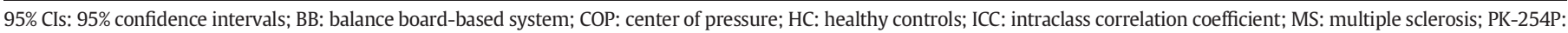
reference standard force platform; SD: standard deviation; SEM: standard error of the measurement. 
Table 3

Summary of study results in terms of concurrent validity (only in patient group, $\mathrm{n}=90$ )

\begin{tabular}{|c|c|c|c|c|c|}
\hline \multicolumn{6}{|c|}{ Concurrent validity } \\
\hline \multicolumn{2}{|c|}{ Self-reporting of accidental falls } & \multirow{2}{*}{$\begin{array}{l}\text { Previous month } \\
10(11 \%)\end{array}$} & \multirow{2}{*}{$\begin{array}{l}\text { Previous } 3 \text { months } \\
17(19 \%)\end{array}$} & Previous 6 months & \multirow{2}{*}{$\begin{array}{l}\text { Previous } 12 \text { months } \\
30(33 \%)\end{array}$} \\
\hline No. of patients & & & & $23(26 \%)$ & \\
\hline \multirow[t]{2}{*}{ AUC (95\% CIs) } & PK-254P & $0.759(0.649$ to 0.836$)$ & $0.758(0.657$ to 0.842$)$ & $0.773(0.672$ to 0.854$)$ & $0.766(0.655$ to 0.849$)$ \\
\hline & $\mathrm{BB}$ & $0.751(0.640-0.817)$ & $0.748(0.647$ to 0.834$)$ & 0.761 (0.658 to 0.844$)$ & 0.759 (0.657 to 0.843$)$ \\
\hline \multicolumn{2}{|c|}{ Difference (95\% CIs) } & $0.008(-0.065$ to 0.098$)$ & $0.010(-0.091$ to 0.115$)$ & $0.013(-0.011$ to 0.016$)$ & $0.007(-0.057$ to 0.072$)$ \\
\hline \multicolumn{2}{|c|}{ p-value } & 0.826 & 0.815 & 0.710 & 0.847 \\
\hline
\end{tabular}

BB: balance board-based system; PK-254P: reference standard force platform.

In $\mathrm{HC}$ group, the between-device reliability was just adequate (ICC $=0.467,95 \% \mathrm{CI}: 0.173-0.796)$, while test-retest reliability was excellent (ICC $=0.885,95 \% \mathrm{CI}: 0.730-0.976$ ), with SEM of 66 and $31 \mathrm{~mm}$, respectively (see also Table 2 ).

\subsection{Concurrent validity in patients with MS}

Comparison of ROC curves showed no significant between-device difference in detecting the occurrence of accidental falls at different timeframes, with AUC from 0.758 to 0.766 (PK-254P) versus AUC from 0.748 to 0.761 (BB), all $p$-values $>0.7$ (Table 3 ).

This latter suggests that the two device had similar performance in discriminating fallers and non-fallers (self-reported) among MS people.

\section{Discussion}

The low-expensive, portable, and user-friendly BB-based system presents acceptable reliability with respect to the reference standard PK-254P, and even a better test-retest reliability when equipped with the custom-written "We-Measure" software in both patients with MS and control group. The lower between-device reliability observed in HC than MS group (47\% versus 67\%) supports the suggestion that the BB-based system is useful only in situations where lower accuracy and precision may be acceptable [17]. In fact, the main technical limitation of $\mathrm{BB}$ encompasses its low-resolution measurements compared to laboratory-grade force platforms, as suggested by previously published studies [17,23], and by the presence of some outliers at extremities of measurements in our study groups, especially in subjects who had the smallest and the largest amount of postural sway. Nevertheless, the lack of significant between-device differences in discriminating fallers and non-fallers among the patient group supports its concurrent validity.

So far, studies exploring the repeatability of BB-based measurements of postural sway have provided conflicting results. Leach and coll. [23] report a low variability across multiple items, i.e. different BB may provide consistent measurements. On the contrary, by comparing the measurement performance of lightly and heavily used BBs, Bartlett and coll. [17] found that the total uncertainty of force measurements exceeded the recommended level uncertainty [15]. However, the repeatability of a single measurement within a single item was better than that between different items, suggesting that the BB is best used for relative measures using the same device, rather than absolute measurement across devices [17].

This implies that BB cannot replace a laboratory-grade force platform in cross-sectional study design, but the excellent test-retest reliability found in our study (84-88\%) may potentially encourage its use in longitudinal study. However, experimental data supporting the over time stability of BB-generated outputs even within the same device should be produced before the BB may be reliably used as a measurement tool [16,24,25].

\section{Conclusion}

The present research may potentially represent the next step towards the implementation of a custom-written software capable to provide a low-cost, portable static posturography that may be reliably used for multicenter purposes.

\section{Ethical standards}

This study was conducted in accordance with the International Conference on Harmonization Guidelines of Good Clinical Practice and the ethical standards laid down in the 1964 Declaration of Helsinki and its later amendments.

The study protocol was approved by the Ethical Committee of Sapienza University; each participant provided written informed consent before any study-related procedure.

\section{Funding source}

This research was carried out using information collected during normal patient care, and extra time was spent in data analysis and interpretation; no external source of funding or specific grant from any funding agency in the public, commercial, or not-for-profit sectors was received.

\section{Conflict of interest}

None declared.

No support was received from Nintendo.

\section{Disclosures}

LP has received research grants from Associazione Italiana Sclerosi Multipla, consulting fees from Biogen and Novartis; speaker honoraria and travel grants from Biogen, Genzyme, Novartis and Teva. The other authors have nothing to disclose.

\section{Acknowledgments}

We would like to thank Prof. Carlo Pozzilli for his advice, support and intellectual guidance.

\section{References}

[1] L. Prosperini, C. Pozzilli, The clinical relevance of force platform measures in multiple sclerosis: a review, Mult. Scler. Int. 2013 (2013) 756564.

[2] G.M. Ramdharry, et al., De-stabilizing and training effects of foot orthoses in multiple sclerosis, Mult. Scler. 12 (2006) 219-226.

[3] V. Emmerik, et al., Postural control in women with multiple sclerosis: effects of task, vision and symptomatic fatigue, Gait Posture 32 (2010) 608-614.

[4] D. Cattaneo, J. Jonsdottir, Sensory impairments in quiet standing in subjects with multiple sclerosis, Mult. Scler. 15 (2009) 59-67. 
[5] J.M. Huisinga, J.M. Yentes, M.L. Filipi, N. Stergiou, Postural control strategy during standing is altered in patients with multiple sclerosis, Neurosci. Lett. 524 (2) (2012) 124-128.

[6] R.A. Clark, A.L. Bryant, Y. Pua, P. McCrory, K. Bennell, M. Hunt, Validity and reliability of the Nintendo Wii Balance Board for assessment of standing balance, Gait Posture 31 (2010) 307-310.

[7] B. Hubbard, D. Pothier, C. Hughes, J. Rutka, A portable, low-cost system for posturography: a platform for longitudinal balance telemetry, J. Otolaryngol. Head Neck Surg 41 (Suppl. 1) (2012) S31-S35.

[8] D.S. Park, G. Lee, Validity and reliability of balance assessment software using the Nintendo Wii balance board: usability and validation, J. Neuroeng. Rehabil. 11 (2014) 99.

[9] D.J. Goble, B.L. Cone, B.W. Fling, Using the Wii Fit as a tool for balance assessment and neurorehabilitation: the first half decade of "Wii-search", J. Neuroeng. Rehabil. 11 (2014) 12.

[10] W.D. Chang, W.Y. Chang, C.L. Lee, C.Y. Feng, Validity and reliability of wii fit balance board for the assessment of balance of healthy young adults and the elderly, J. Phys. Ther. Sci. 25 (2013) 1251-1253.

[11] K.J. Bower, J.L. McGinley, K.J. Miller, R.A. Clark, Instrumented static and dynamic balance assessment after stroke using Wii Balance Boards: reliability and association with clinical tests, PLoS One 9 (2014), e115282.

[12] P. Scaglioni-Solano, L.F. Aragón-Vargas, Validity and reliability of the Nintendo Wii Balance Board to assess standing balance and sensory integration in highly functional older adults, Int. J. Rehabil. Res. 37 (2014) 138-143.

[13] F. Sgrò, G. Monteleone, M. Pavone, M. Lipoma, Validity Analysis of Wii Balance Board versus baropodometer platform using an open custom integrated application, AASR Procedia 8 (2014) 22-29.

[14] P.E. Jeter, J. Wang, J. Gu, M.P. Barry, C. Roach, M. Corson, L. Yang, G. Dagnelie, Intrasession test-retest reliability of magnitude and structure of center of pressure from the Nintendo Wii Balance Board ${ }^{\mathrm{TM}}$ for a visually impaired and normally sighted population, Gait Posture 41 (2015) 482-487.
[15] Scoppa, et al., Clinical stabilometry standardization: basic definitions-acquisition interval-sampling frequency, Gait Posture 37 (2013) 290-292.

[16] G. Pagnacco, E. Oggero, C.H. Wright, Biomedical instruments versus toys: a preliminary comparison of force platforms and the Nintendo wii balance board, Biomed. Sci. Instrum. 47 (2011) 12-17.

[17] H.L. Bartlett, L.H. Ting, J.T. Bingham, Accuracy of force and center of pressure measures of the Wii Balance Board, Gait Posture 39 (2014) 224-228.

[18] J.H. Sheldon, The effect of age on the control of sway, Gerontol. Clin. 5 (1963) 129-138.

[19] M. Salavati, M.R. Hadian, M. Mazaheri, H. Negahban, I. Ebrahimi, S. Talebian, Testretest reliability of center of pressure measures of postural stability during quiet standing in a group with musculoskeletal disorders consisting of low back pain, anterior cruciate ligament injury and functional ankle instability, Gait Posture 29 (2009) 460-464.

[20] L. Prosperini, D. Fortuna, C. Giannì, L. Leonardi, C. Pozzilli, The diagnostic accuracy of static posturography in predicting accidental falls in people with multiple sclerosis, Neurorehabil. Neural Repair 27 (2013) 45-52.

[21] P. Schubert, M. Kirchner, D. Schmidtbleicher, C.T. Haas, About the structure of posturography: sampling duration, parametrization, focus of attention (part I), J. Biomed. Sci. Eng. 5 (2012) 496-507.

[22] J. Lee, D. Koh, C.N. Ong, Statistical evaluation of agreement between two methods for measuring a quantitative variable, Comput. Biol. Med. 19 (1989) 61-70.

[23] J.M. Leach, M. Mancini, R.J. Peterka, T.L. Hayes, F.B. Horak, Validating and calibrating the Nintendo Wii balance board to derive reliable center of pressure measures, Sensors (Basel) 14 (2014) 18244-18267.

[24] T.A. Stoffregen, B.G. Bardy, When the WBB is useful, and when it isn't, Gait Posture 39 (2014) 1154.

[25] G. Pagnacco, M.W. Bundle, F.R. Carrick, C.H. Wright, E. Oggero, Letter to the editor: on "validity and reliability of the Nintendo Wii Balance Board for assessment of standing balance" by R.A. Clark et al. [Gait \& Posture 31 (2010) 307-310]: are the conclusions stated by the authors justified? Gait Posture 39 (2014) 1150-1151. 